\title{
Primary multi-drug resistant tuberculosis presented as lymphadenitis in a patient without HIV infection
}

\author{
S.M. Mirsaeidi, P. Tabarsi, M.O. Edrissian, M. Amiri, P. Farnia, \\ S.D. Mansouri, M.R. Masjedi, A.A. Velayati
}

ABSTRACT: Primary multi-drug resistant tuberculosis presented as lymphadenitis in a patient without HIV infection. S.M. Mirsaeidi, P. Tabarsi, M.O. Edrissian, M. Amiri, P. Farnia, S.D. Mansouri, M.R. Masjedi, A.A. Velayati.

Primary multi-drug resistant extrapulmonary tuberculosis is an uncommon form of the disease, but it seems that by increasing the number drug resistant tuberculosis around the world, the number of cases of primary multidrug resistant tuberculosis with extrapulmonary presentation also is going to rise. In this report, we describe a 19year old, HIV negative man with primary multi-drug resistant TB lymphadenitis, presented with cervical lym- phadenopathy and sinus discharge at the site of involved lymph nodes. The Acid Fast Bacilli (AFB) smear of sputum was negative but the AFB smear of discharged fluid as well as the excisional biopsy of the lymph nodes confirmed the $M$. tuberculosis infection. The patient underwent the treatment with a combination of isoniazide, clofazimine, pyrazinamide, ofloxacin and amikacin with promising results. By increasing the number of drug resistant tuberculosis patients around the world, appropriate diagnosis and treatment of different presentations of the disease need a special attention.

Monaldi Arch Chest Dis 2004; 61: 4, 244-247.

Keywords: HIV Antibodies, Iran, Lymphadenitis, Tuberculosis, Multidrug-Resistant.

National Research Institute of Tuberculosis and Lung Diseases, Shaheed Beheshti University of Medical Sciences, Tehran, Iran.

Correspondence: Mirsaeidi SM, National Research Institute of Tuberculosis and Lung Diseases, Shaheed Beheshti University of Medical Sciences, Niavaran,Darabad,19556,Tehran,Iran; e-mail:mmirsaeidi@nritld.ac.ir

\section{Introduction}

Tuberculosis (TB) is an infectious disease with various clinical presentations. Due to the fact that the proportion of TB cases caused by drug-resistant strains has increased around the world in recent years, appropriate diagnosis and management of these patients is vital in controlling the disease.

Among the immunocompetent primary multidrug resistant TB patients, extra pulmonary presentation of TB is not very common and experience in the management of drug resistant extrapulmonary TB is limited $[1,2]$. In this report, we presented a HIV-seronegative primary multi-drug resistant TB patient with lymphadenitis presentation.

\section{Case presentation}

A 19 year old Afghan man presented to the outpatient clinic of Tuberculosis in Dr. Massih Daneshvari Hospital with a lump in the right submandibular region, mild anorexia and weight loss. The mass appeared on his neck 2 years ago with a progressive trend. The patient was admitted to the TB ward of the hospital for further evaluation. $\mathrm{He}$ had not received any specific management for his problem up to the time of admission. He had a positive history of non-specific fever and chills but he did not mention any history of dyspnea, chest pains, coughs, sputum production and night sweating. He is a non-smoker and the past medical history for any specific disease was negative. He did not mention the history of any close contact with a recognized TB patient. He immigrated to Iran 4 years ago and he was working as a construction worker.

In the physical examination, a movable prominent palpable mass with $2 \times 3 \mathrm{~cm}$ diameters was observed in left submandibular region along with three less prominent lymph nodes in left anterior lymphatic chain of the neck. No palpable nodes were found in axilla or supraclavicular areas. The physical examination of other organs was normal. Hematological and biochemical parameters were normal and a Tuberculin skin test (with PPD) for Mycobacterium tuberculosis showed a 14 millimeter induration. Microscopical examination of sputum smear was negative for Mycobacterium tuberculosis bacilli. An Anti-HIV antibody (ELISA) test was negative. Immunoglublin measures, $\mathrm{Ni}$ troblue tetrazolium test and adenosine deaminase test were normal. The flow cytometery result was normal except for a slight decrease in CD3/CD4 percent (table 1). The patient also underwent the 
Table 1. - The results of the laboratory tests of patient

\begin{tabular}{|c|c|c|c|}
\hline & Result & & Result \\
\hline \multicolumn{2}{|l|}{ Hematological variables: } & \multicolumn{2}{|l|}{ Immunologic variables \& other TB-related laboratory tests } \\
\hline Red cell count (per $\mathrm{mm}^{3}$ ) & $4,350,000$ & Immunoglobulin G (SRID) (mg/dl) & 1,700 \\
\hline Hematocrit (\%) & 35.5 & Immunoglobulin A (SRID) (mg/dl) & 250 \\
\hline Hemoglobin (g/dl) & 11.7 & Immunoglobulin M (SRID) (mg/dl) & 150 \\
\hline Mean corpuscular volume $\left(\mu \mathrm{m}^{3}\right)$ & 81.6 & Immunoglobulin G (ELISA) (mg/dl) & 106 \\
\hline White-cell count (per $\mathrm{mm}^{3}$ ) & 6,900 & T helper lymphocytes & \\
\hline Differential count $(\%)$ & & $(\mathrm{CD} 3 / \mathrm{CD} 4)(\%)$ (Normal range: 28.5-60.5) & 24 \\
\hline Neutrophils & 72 & Anti-HIV antibody (ELISA) & Negative \\
\hline Lymphocytes & 24 & Tuberculin skin Test & $14 \mathrm{~mm}$ \\
\hline Monocytes & 1 & AFB smear of sputum (repeated 4 times) & Negative \\
\hline Eosinophils & 3 & AFB smear of lymph node tissue & Positive \\
\hline Platelet count (per $\mathrm{mm}^{3}$ ) & 349,000 & AFB smear of discharged fluid from Lymphadenitis & Positive \\
\hline Prothrombin time (sec) (activity 100\%) & 13 & Mycobacterial culture and DST & \\
\hline \multirow[t]{2}{*}{ Erythrocyte sedimentation rate ( $\mathrm{mm} /$ hour) } & 32 & M.tuberculosis (confirmed by PCR) & ant to $\mathrm{H}+\mathrm{R}+\mathrm{S}+\mathrm{E}$ \\
\hline & & Nitroblue tetrazolium test $(\%)$ & 100 \\
\hline Blood Chemical Variables: & & Adenosine deaminase test (negative if $<45 \mathrm{U} / \mathrm{l}$ ) & 42 \\
\hline Fasting blood sugar $(\mathrm{mg} / \mathrm{dl})$ & 108 & & \\
\hline Urea (mg/dl) & 28 & & \\
\hline Creatinine (mg/dl) & 0.7 & & \\
\hline Sodium $(\mathrm{mEq} / \mathrm{dl})$ & 139 & Urine analysis: & \\
\hline Potassium $(\mathrm{mEq} / \mathrm{dl})$ & 4.2 & Sugar & Negative \\
\hline Aspartate aminotransferase (IU/l) & 25 & White blood cell & $1-2$ \\
\hline Alanine aminotransferase(IU/l) & 17 & Red blood cell & $0-1$ \\
\hline Alkaline phosphatase (IU/l) & 270 & Hemoglobin & Negative \\
\hline Billirubin Total (mg/dl) & 0.4 & Urine culture & Negative \\
\hline
\end{tabular}

excisional biopsy of the mass in his neck which the result of pathological examination showed chronic necrotizing granulomatosis inflammation consistent with TB. The Acid Fast Bacilli smear of the specimen was positive for Mycobacterium tuberculosis. The specimen was also sent for mycobacterial and mycological culture and antibiogram. Mycology culture of the specimen was negative. The results of the laboratory tests are summarised in table 1. The chest X-ray was normal (fig. 1), but the computed tomography of chest with intravenous contrast showed calcified hilar and subcarinal nodes and a small pleural tag in posterior segment of right upper lobe (fig. 2). Computed tomography of neck revealed numerous peripherally enhancing lymph nodes (fig. 3). By diagnosing the TB infection in the patient, the standardised treatment regimen [3] commenced for the patient (patient's weight was $50 \mathrm{~kg}$ ) as follows: isoniazid 250

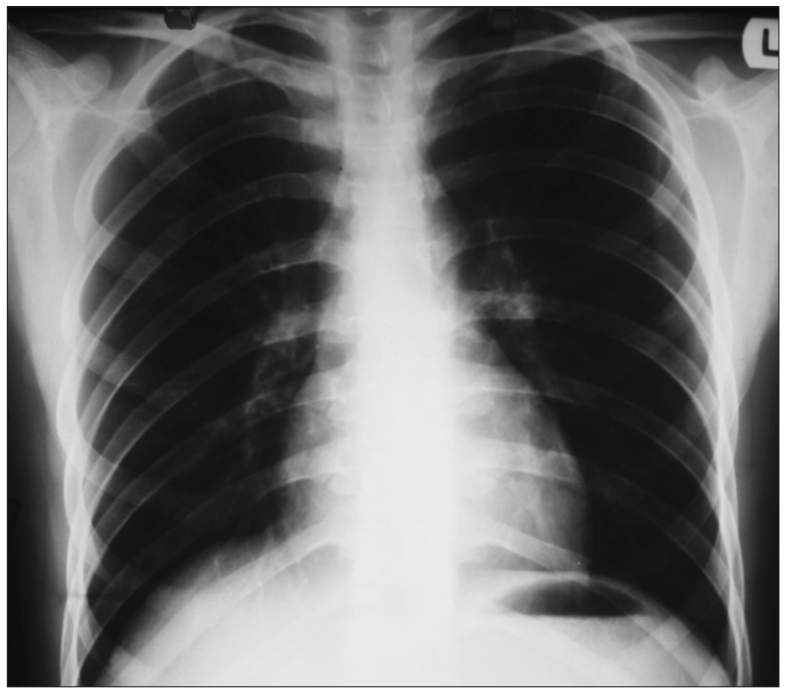

Fig. 1. - The chest X-ray was normal. 
$\mathrm{mg}$ daily, rifampicin $600 \mathrm{mg}$ daily, pyrazinamide $1250 \mathrm{mg}$ daily and etambutol $1000 \mathrm{mg}$ daily.

Two months after beginning the treatment, not only was no improvement observed in the patient's condition, but also several lymphadonpathies had appeared in the posterior and anterior lymphatic chains in the patient's neck with fistulisation in both sides and thick purulent discharge (fig. 4). The AFB

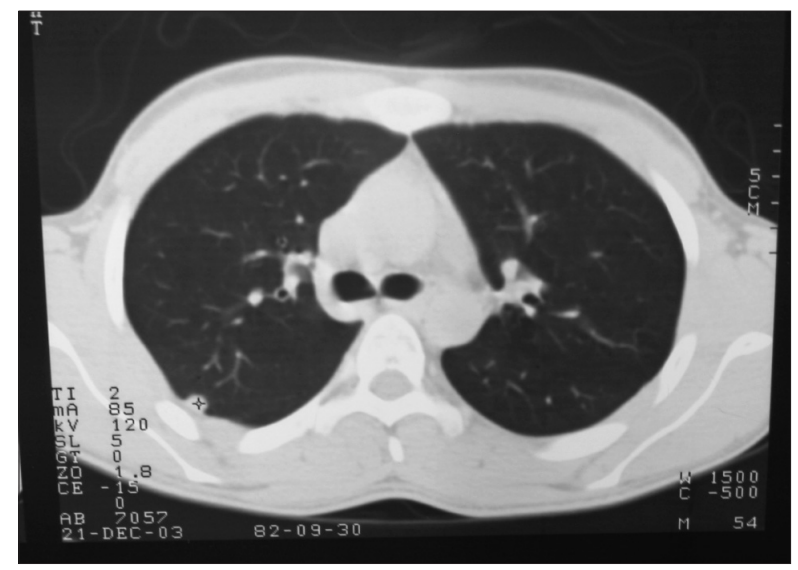

Fig. 2. - Computed tomography of chest with intravenous contrast. It shawed a small pleural tag, in posterior segment of right upper lobe.

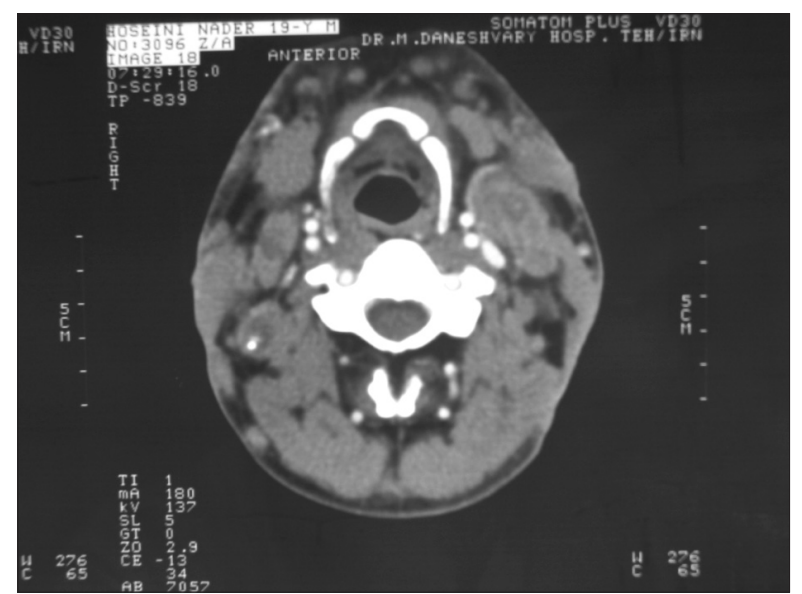

Fig. 3. - Computed tomography of neck; revealed numerous pripherally enhancing lymphnodes. smear of discharged fluid and polymerase chain reaction of the specimen confirmed the infection by Mycobacterium tuberculosis bacilli. At the time, the results of culture in Löwenstein-Jensen (LJ) medium and anti-TB drug susceptibility testing (DST) performed by proportion method showed a multi-drug resistance $M$. Tuberculosis which was resistant to isoniazid $(\mathrm{H})$, rifampicin $(\mathrm{R})$ streptomycin (S), and ethambutol (E). Repeating the culture and DST confirmed the previous results, later. The patient was re-interviewed by different interviewers and no history of previous anti-TB therapy was taken. At this time, repeating the physical examination revealed developing of a $1 \times 1 \mathrm{~cm}$ lymphadenopathy in the left retroauricular region as well as a 1x1 cm right auxiliary lymph node and $1 \times 1 \mathrm{~cm}$ right inguinal lymph node. The treatment strategy was changed by diagnosing tuberculosis with primary drug resistance and the following regimen considered for the patient: isoniazide $500 \mathrm{mg}$ daily, pyrazinamide $1500 \mathrm{mg}$ daily, ofloxacin 600 $\mathrm{mg}$ daily, amikacin $500 \mathrm{mg}$ daily and clofazimine $200 \mathrm{mg}$ daily.

Two weeks after beginning the new treatment, lymphadenitis and fistula discharge subsided obviously (fig. 4) and a month after the treatment, the result of the smear of discharged fluid changed to negative. We plan to continue the treatment for at least 18 months with precise monitoring and follow up.

\section{Discussion}

Drug resistant TB has become a major public health problem since early 1990's globally $[4,5]$. The prevalence of new cases of multidrug resistant TB in Iran was estimated 5.0 percent; however this prevalence is 48.2 percent among previously treated cases [6]. Drug-resistant TB was significantly higher $(\mathrm{p}<0.05)$ in the foreign-born TB patients than in indigenous patients in Iran [7]. Cervical lymphadenitis is a common extrapulmonary manifestation of TB; nevertheless, it seems that the primary multidrug resistant extrapulmonary TB is an uncom-
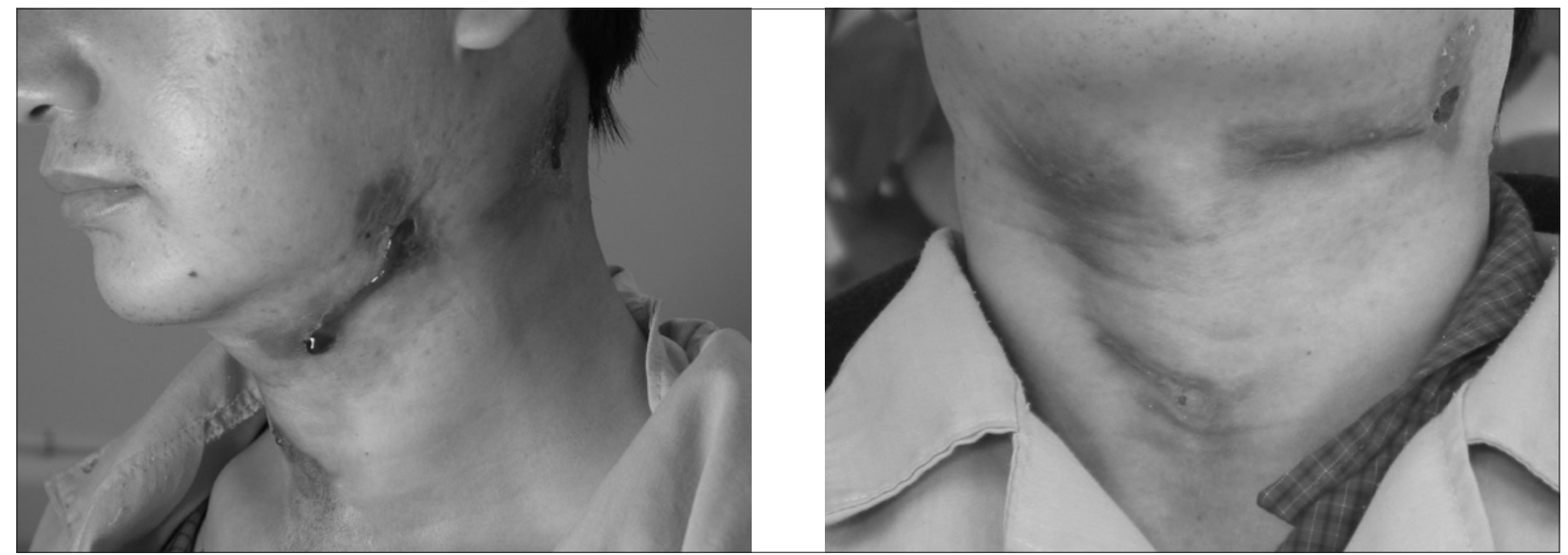

Fig. 4. - Before and after treatment photos of the patient neck. 
mon form of the disease [1]. The reported patient is the first immunocompetent patient with primary multi-drug resistant TB in the National Research Institute of Tuberculosis and Lung Disease that is the main referral center for TB in Iran and else where (as our knowledge). The case does not report any previous exposure to TB. In lymphadenitis TB, less than $20 \%$ of patients have a history of previous exposure to TB patients [8]. The chest-X-ray in this patient was normal and the primary site of TB was found in his lungs by performing computer tomography scan. This is not a surprising finding because only in $24-46 \%$ of patients with TB lymphadenitis, radiographic abnormalities consistent with TB can be observed $[9,10]$.

While the treatment response in cases with multi-drug resistance had a significantly higher failure rate than those who were susceptible [2, 11], we have had a desirable response to treatment with isoniazide, clofazimine, pyrazinamide, ofloxacin and amikacin in our case with the changing of the discharged fluid smear to negative, significant subsiding the lymphadenitis and general well being. Nevertheless, this may be due to the lack of serious involvement of vital organs like the lung in the patient.

By increasing the number of the drug resistant TB cases around the world, facing the various manifestation of this type of the disease, including the primary multi-drug resistant TB with extrapulmonary presentation is expected to be higher than before [1], which brings more emphasis on rapid and unambiguous diagnosis and suitable management of the disease as a consequence.

\section{References}

1. Friedman LN, Tuberculosis: current concepts and treatment, CRC press LLC, 2nd ed., 2001; 352.

2. Long R. Drug-resistant tuberculosis, CMAJ 2000 Aug 22; 163 (4): 425-8.

3. World Health Organisation. Treatment of tuberculosis Guidelines for national programmes, $3^{\text {rd }}$ ed. Geneva: WHO, 2003.

4. Anti-tuberculosis drug resistance in the world: The WHO/IUATLD Global Project on Anti-tuberculosis Drug Resistance Surveillance 1994-1997. Geneva: World Health Organization, 1997.

5. Pablos-Mendez A, Raviglione MC, Laszlo A, et al. Global surveillance for antituberculosis-drug resistance. 1994-1997. N Engl J Med 1998; 338: 1641-9.

6. Espinal MA, Laszlo A, Simonsen L, Boulahbal F, et al. Global trends in resistance to antituberculosis drugs. World Health Organization-International Union against Tuberculosis and Lung Disease Working Group on Anti-Tuberculosis Drug Resistance Surveillance. $N$ Engl J Med 2001 Apr 26; 344 (17): 1294-303.

7. Espinal MA, Laszlo A, Simonsen L, Boulahbal F, et al. Anti-tuberculosis drug resistance in the world, report No.2, prevalence and trends, World Health Organization-International Union against Tuberculosis and Lung Disease Working Group on Anti-Tuberculosis Drug Resistance Surveillance, communicable diseases, WHO 2000: 73.

8. Kent DC. Tuberculosis lymphadenitis: not a localized disease process. Am J Med Sci 1967 Dec; 254 (6): 866-74.

9. Shikhani A H, Hadi U M, Muffarij A A, et al. Mycobacterial cervical lymphadenitis. Ear Nose Throat $J$ 1989 Sep; 68 (9): 660, 662-6, 668-72.

10. Lee K C, Tami T A, Lalwani A K, et al. Comtemporary management of cervical TB, Laryngoscope 1992 Jan; 102 (1): 60-4.

11. Espinal MA, Kim SJ, Suarez PG, et al. Standard shortcourse chemotherapy for drug-resistant tuberculosis: treatment outcomes in 6 countries, JAMA 2000 May 17; 283 (19): 2537-45.

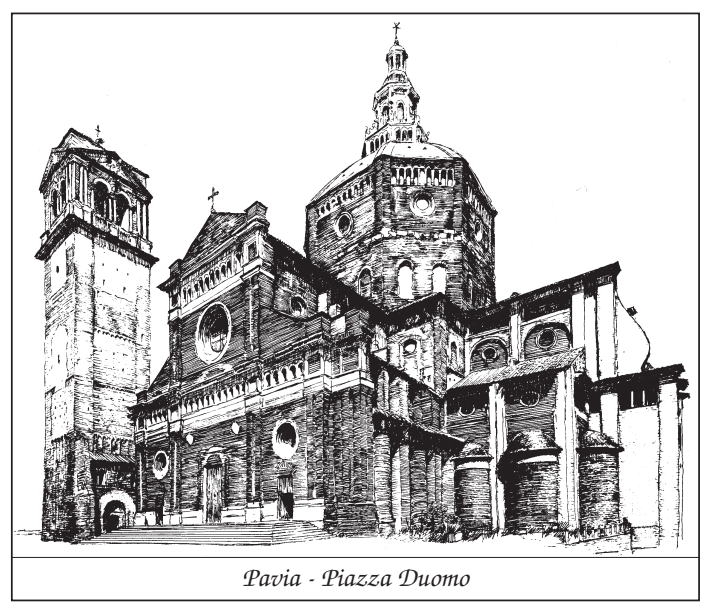

